JOURNAL OF THE

AMERICAN MATHEMATICAL SOCIETY

Volume 19, Number 1, Pages 251-263

S $0894-0347(05) 00504-7$

Article electronically published on August 24, 2005

\title{
SHARP TRANSITION BETWEEN EXTINCTION AND PROPAGATION OF REACTION
}

\author{
ANDREJ ZLATOŠ
}

\section{INTRODUCTION}

In the present paper we consider the reaction-diffusion equation

$$
T_{t}=\Delta T+f(T)
$$

in the cylinder $\mathbb{R} \times \Omega$ where $\Omega$ is a domain in $\mathbb{R}^{n-1}$, with Neumann boundary conditions on $\mathbb{R} \times \partial \Omega$. The non-linear reaction function $f$ is assumed to be Lipschitz continuous with $f(0)=f(1)=0$ and the initial datum $T_{0}$ is between 0 and 1 .

We will treat the case when $T_{0}$ is independent of the transversal variable $y \in \Omega$, and so (1.1) becomes

$$
T_{t}=T_{x x}+f(T)
$$

with $x \in \mathbb{R}$. This equation has been extensively studied in mathematical, physical and other literature, starting with the pioneering works of Fisher [7] and Kolmogorov, Petrovskii, Piskunov 11. In these papers (1.2) was used to describe the propagation of advantageous genes in a population. The main object of study in these and many subsequent works was the existence and stability of traveling fronts for (1.2) and (1.1). In recent years most of the results have been extended to include an advection term $u \cdot \nabla T$ in (1.1), and we refer to the reviews [2, 16] for an extensive bibliography.

The above equations are used to model more than just population genetics phenomena. When $f(\theta)>0$ for $\theta \in(0,1)$, then $f$ is a combustion non-linearity and (1.1) / (1.2) model an exothermic chemical reaction in an infinite tube with a zero heat-loss boundary, in particular, flame propagation in a premixed combustible gas without advection (see Zel'dovich and Frank-Kamenetskii [17]). In this setting T is the normalized temperature taking values in $[0,1]$. We note that (1.1) is usually obtained from a system involving both the temperature and the concentration of the reactants after the simplifying assumption of equal thermal and material diffusivities.

A special case of positive $f$, used particularly in chemical and biological literature, is the KPP type with $f^{\prime \prime}(\theta) \leq c<0$ [11. In combustion models the non-linearity is often considered to be of Arrhenius type with slow reaction rates at low temperatures, modeled by $f(\theta)=e^{-A / \theta}(1-\theta)$. This case is often approximated

Received by the editors April 15, 2005.

2000 Mathematics Subject Classification. Primary 35K57; Secondary 35K15.

Key words and phrases. Reaction-diffusion equation, quenching, ignition non-linearity, bistable non-linearity.

(C)2005 American Mathematical Society Reverts to public domain 28 years from publication 
by an ignition non-linearity $f$ satisfying $f(\theta)=0$ for $\theta \in\left[0, \theta_{0}\right]$ and $f(\theta)>0$ for $\theta \in\left(\theta_{0}, 1\right)$, with $\theta_{0} \in(0,1)$ the ignition temperature.

The last prominent case is bistable non-linearity with $f(\theta)<0$ for $\theta \in\left(0, \theta_{0}\right)$ and $f(\theta)>0$ for $\theta \in\left(\theta_{0}, 1\right)$, where one usually assumes $\int_{0}^{1} f(\theta) d \theta>0$. This has been used to model signal propagation along bistable transmission lines, in particular, nerve pulse propagation [12. In a biological context it is also called heterozygote inferior (see Aronson and Weinberger [1]).

In this paper we will consider all the above types. Our interest here will not be in the question of traveling fronts, but in extinction of reaction - quenching of flames. We will therefore assume the initial datum $T_{0}(x)$ for (1.2) to be compactly supported and will want to know when

$$
\|T(t, \cdot)\|_{\infty} \rightarrow 0 \text { as } t \rightarrow \infty .
$$

For the sake of simplicity we will restrict ourselves to the case of $T_{0}$ being the characteristic function of an interval,

$$
T_{0}(x) \equiv \chi_{[-L, L]}(x),
$$

and study how long-time behavior of $T$ depends on $L$. The methods in this paper allow one to treat some other increasing 1-parameter families of initial conditions as well.

Thus, we will study the competition of reaction and diffusion. The former helps increasing the temperature, whereas the latter (together with the compactness of the support of the initial datum) works towards the extinction of the flame. This question was originally addressed forty years ago by Kanel' $[9$ who considered the case of ignition non-linearity and proved that if the initial datum is large enough, then reaction wins, whereas if it is small, then diffusion manages to quench the flame. More precisely, when $T$ solves (1.2), (1.4) and $f$ is of ignition type, Kanel proved that there are two length scales $L_{0}, L_{1}$ such that

$$
\begin{aligned}
& T(t, x) \rightarrow 0 \text { as } t \rightarrow \infty \text { uniformly in } x \in \mathbb{R} \text { if } L<L_{0}, \\
& T(t, x) \rightarrow 1 \text { as } t \rightarrow \infty \text { uniformly on compacts if } L>L_{1} .
\end{aligned}
$$

This has been extended to the case of bistable $f$ by Aronson and Weinberger [1]. Both results also hold when (1.4) is replaced by

$$
T_{0}(x) \equiv \alpha \chi_{[-L, L]}(x)
$$

for any $\alpha>\theta_{0}$, with $L_{0}$ and $L_{1}$ depending on $\alpha$ (in the ignition case this follows from [9], in the bistable case it was proved by Fife and McLeod [6]). A natural question arises: does $L_{0}$ equal $L_{1}$ ? If this is true and if one could determine the behavior of $T$ as $t \rightarrow \infty$ when $L=L_{0}$, then one would be able to provide the complete "phase portrait" for the PDE (1.2) with respect to a 1-parameter family of initial conditions.

Since these early works, particularly in recent years, several authors have studied quenching for (1.1). The above results have been extended to the case when (1.1) includes an advection term $u \cdot \nabla T$, with $u$ a shear or periodic flow (see [13, 15), even for certain combustion non-linearities [18]. Quenching of large initial data by large amplitude shear and cellular flows has been studied in [4, 5, 10, 18. However, the question whether $L_{0}=L_{1}$ remained open even in the simplest case of (1.2). The following two results provide the answer, including the treatment of the critical case $L=L_{0}$. 
The first of them holds for ignition and combustion non-linearities.

Theorem 1. Let $\theta_{0} \in[0,1)$ and $f:[0,1] \rightarrow \mathbb{R}$ be Lipschitz with $f(\theta)=0$ when $\theta \in$ $\left[0, \theta_{0}\right], f(\theta)>0$ when $\theta \in\left(\theta_{0}, 1\right)$, and $f(1)=0$. If $\theta_{0}>0$, then assume in addition that $f$ is non-decreasing on $\left[\theta_{0}, \theta_{0}+\delta\right]$ for some $\delta>0$. Let $T:[0, \infty) \times \mathbb{R} \rightarrow[0,1]$ solve

$$
\begin{aligned}
& T_{t}=T_{x x}+f(T), \\
& T_{0}(x) \equiv \chi_{[-L, L]}(x) .
\end{aligned}
$$

Then there is $L_{0} \geq 0$ such that

(i) if $L<L_{0}$, then $T \rightarrow 0$ uniformly on $\mathbb{R}$ as $t \rightarrow \infty$;

(ii) if $L=L_{0}$, then $T \rightarrow \theta_{0}$ uniformly on compacts as $t \rightarrow \infty$;

(iii) if $L>L_{0}$, then $T \rightarrow 1$ uniformly on compacts as $t \rightarrow \infty$.

Remark. The possibility of $L_{0}=0$ (the so-called hair-trigger effect) cannot be excluded when $\theta_{0}=0$. More precisely, using results from 18 one can show that $L_{0}=0$ when $f(\theta) \geq c \theta^{p}$ for some $p<3$ and all small $\theta$, but $L_{0}>0$ when $f(\theta) \leq c \theta^{p}$ for some $p>3$ and all small $\theta$. Note also that if $\theta_{0}=0$, then the convergence in (ii) is as in (i) - uniform on $\mathbb{R}$.

Our second result holds for bistable non-linearities. We define $\theta_{2} \in\left(\theta_{0}, 1\right)$ by $\int_{0}^{\theta_{2}} f(\theta) d \theta=0$ and let $U$ be the unique function solving $0=U^{\prime \prime}+f(U)$ with $U(0)=\theta_{2}$ and $U^{\prime}(0)=0$. Then $U$ is an even function and we will show in the proof of the following theorem that it is positive on $\mathbb{R}$, decreasing to 0 on $(0, \infty)$, and bell-shaped.

Theorem 2. Let $\theta_{0} \in(0,1)$ and $f:[0,1] \rightarrow \mathbb{R}$ be Lipschitz with $f(0)=f\left(\theta_{0}\right)=$ $f(1)=0, f(\theta)<0$ when $\theta \in\left(0, \theta_{0}\right)$, and $f(\theta)>0$ when $\theta \in\left(\theta_{0}, 1\right)$. Assume also that $\int_{0}^{1} f(\theta) d \theta>0$ and $U$ is as above. Let $T:[0, \infty) \times \mathbb{R} \rightarrow[0,1]$ solve the problem (1.6). Then there is $L_{0}>0$ such that

(i) if $L<L_{0}$, then $T \rightarrow 0$ uniformly on $\mathbb{R}$ as $t \rightarrow \infty$;

(ii) if $L=L_{0}$, then $T \rightarrow U$ uniformly on $\mathbb{R}$ as $t \rightarrow \infty$;

(iii) if $L>L_{0}$, then $T \rightarrow 1$ uniformly on compacts as $t \rightarrow \infty$.

Remark. Both theorems can be extended to some other increasing families of initial conditions, in particular, to (1.5) with $\alpha>\theta_{0}$.

The crux of the proofs of both theorems will be to show that there is a single $L$ for which $T$ does not converge to 0 or 1 at $x=0$ as $t \rightarrow \infty$. In Theorem 1 this will be achieved with the help of Lemma 4 by comparing solutions of (1.6) for two different initial conditions at differently rescaled times. In Theorem 2 it will follow from a detailed analysis of the large time behavior of $T$ when the above limit is not 0 or 1 , and an application of the comparison principle.

We note here that Theorem 1 is, in a sense, a limiting case of Theorem 2, If one takes $f \rightarrow 0$ on $\left(0, \theta_{0}\right)$ keeping $f$ unchanged on $\left(\theta_{0}, 1\right)$, one has $\theta_{2} \rightarrow \theta_{0}$ and $U \rightarrow \theta_{0}$ on compacts. That is, the bell-shaped solution $U$ from Theorem 2 (ii) converges to the constant solution $\theta_{0}$ from Theorem 1(ii).

The rest of the paper is devoted to the proofs of the two theorems. Section 2 contains preliminary Lemmas 3 and 4 . Sections 3 and 4 prove Theorems 1 and 2 , respectively. 


\section{Preliminary Lemmas}

We start with

Lemma 3. Let $f:[0,1] \rightarrow \mathbb{R}$ be Lipschitz with $f(0)=f(1)=0$. If $T:[0, \infty) \times \mathbb{R} \rightarrow$ $[0,1]$ solves (1.6), then the following hold.

(i) If $|x| \leq|y|$, then $T(t, x) \geq T(t, y)$.

(ii) There is $t_{*}>0$ (possibly $t_{*}=\infty$ ) such that $T(t, 0)$ as a function of $t$ is non-increasing on $\left[0, t_{*}\right)$ and non-decreasing on $\left[t_{*}, \infty\right)$.

(iii) If $f \geq 0$, then there is $\theta_{*} \in[0,1]$ such that $f\left(\theta_{*}\right)=0$ and $T(t, x) \rightarrow \theta_{*}$ as $t \rightarrow \infty$, uniformly on compacts.

Remarks. 1. For sufficiently smooth $f$ this is essentially a result of Kanel' 9 .

2. In the case of (1.5) with $\alpha \in\left(\theta_{0}, 1\right)$, part (ii) has $0<t_{*} \leq t_{* *} \leq \infty$ such that $T(t, 0)$ is non-decreasing on $\left[0, t_{*}\right)$, non-increasing on $\left[t_{*}, t_{* *}\right)$ and non-decreasing on $\left[t_{* *}, \infty\right)$.

Proof. We first assume that $f$ is smooth and briefly recall main points of the proofs of (i) and (ii) from [9]. Let $T^{\varepsilon}$ solve (1.6) but with initial condition $T(0, x) \equiv \chi^{\varepsilon}(x)$ where $\chi^{\varepsilon}$ are smooth, symmetric, decreasing in $|x|$, and converge to $\chi_{[-L, L]}$ in $L^{1}(\mathbb{R})$ as $\varepsilon \rightarrow 0$. Then $T_{x}^{\varepsilon}(0, x) \leq 0$ for $x>0$, and by symmetry $T_{x}^{\varepsilon}(t, 0)=0$. Since

$$
\left(T_{x}^{\varepsilon}\right)_{t}=\left(T_{x}^{\varepsilon}\right)_{x x}+f^{\prime}\left(T^{\varepsilon}\right) T_{x}^{\varepsilon}
$$

the maximum principle gives $T_{x}^{\varepsilon}(t, x) \leq 0$ for $x>0$. Symmetry then yields $T_{x}^{\varepsilon}(t, x) \geq 0$ for $x<0$. Since for any fixed $t>0$ we have $T^{\varepsilon}(t, x) \rightarrow T(t, x)$ uniformly in $x$ as $\varepsilon \rightarrow 0$, this proves (i).

Now let $D^{h}(t, x) \equiv T(t+h, x)-T(t, x)$. By the mean value theorem,

$$
D_{t}^{h}=D_{x x}^{h}+f^{\prime}(S) D^{h}
$$

for some $S=S(t, x)$. Let $\Delta_{h}$ be the set of $(t, x)$ for which $D_{h}(t, x) \leq 0$. Then $\Delta_{h} \cap(\{0\} \times \mathbb{R})=\{0\} \times[-L, L]$. By the maximum principle and symmetry, $\Delta_{h}$ is connected and its sections by lines parallel to the $x$-axis are segments symmetric about the $t$-axis. Therefore there is $0<t_{*}^{h} \leq \infty$ such that $D^{h}(t, 0)<0$ for $t \in\left[0, t_{*}^{h}\right)$ and $D^{h}(t, 0) \geq 0$ for $t \in\left[t_{*}^{h}, \infty\right)$. From $D^{h}(t, x)=D^{h / 2}\left(t+\frac{h}{2}\right)+D^{h / 2}(t)$ we obtain $t_{*}^{h / 2} \in\left[t_{*}^{h}, t_{*}^{h}+\frac{h}{2}\right]$, and (ii) follows with $t_{*} \equiv \lim _{n \rightarrow \infty} t_{*}^{2^{-n}}$.

If $f$ is only Lipschitz, take smooth $f^{\varepsilon}$ such that $\left\|f^{\varepsilon}-f\right\|_{\infty} \leq \varepsilon$ and let $T^{\varepsilon}$ solve (1.6) with $f^{\varepsilon}$ in place of $f$. One can then show that $V^{\varepsilon} \equiv T^{\varepsilon}-T$ satisfies $\left|V^{\varepsilon}(t, x)\right| \leq \frac{\varepsilon}{c}\left(e^{c t}-1\right)$ with $c$ the Lipschitz constant for $f$ (we spell this argument out in the proof of Theorem 1 below). Therefore $T(t, x)=\lim _{\varepsilon \rightarrow 0} T^{\varepsilon}(t, x)$ for all $t$ and $x$, and since (i) and (ii) hold for each $T^{\varepsilon}$, they also hold for $T$.

Finally, assume that $f \geq 0$. By (ii), $\theta_{*} \equiv \lim _{t \rightarrow \infty} T(t, 0)$ is well defined. Let $\Phi$ solve $\Phi_{t}=\Phi_{x x}$ on $\mathbb{R}^{+}$with $\Phi(0, x) \equiv T(0, x)$ and boundary condition $\Phi(t, 0) \equiv$ $T(t, 0)$. Then $\Phi(t, x) \rightarrow \theta_{*}$ as $t \rightarrow \infty$, uniformly on compacts. Since by the comparison principle (see, e.g., [14]) and (i), $\Phi(t, x) \leq T(t, x) \leq T(t, 0)$, the second claim in (iii) follows.

To prove the first claim, assume $f\left(\theta_{*}\right)>0$ and choose $\varepsilon>0$ such that for $\theta \leq \theta_{*}+10 \varepsilon$ we have $f(\theta) \geq \theta-\theta_{*}+2 \varepsilon$. Pick $t_{0}$ such that if $\Phi$ solves $\Phi_{t}=\Phi_{x x}$ on $\mathbb{R}$ with initial condition $\Phi\left(t_{0}, x\right)=T\left(t_{0}, x\right)$, then $\Phi(t, 0) \geq \theta_{*}-\varepsilon$ and $T(t, x) \leq \theta_{*}+\varepsilon$ for $t \in\left[t_{0}, t_{0}+\ln 4\right]$ and $x \in \mathbb{R}$. This is possible thanks to the second claim in (iii). Define

$$
S(t, x) \equiv \theta_{*}-2 \varepsilon+\left(\Phi(t, x)-\theta_{*}+2 \varepsilon\right) e^{t-t_{0}} .
$$


Then $S(t, x) \leq \theta_{*}+10 \varepsilon$ for $t \in\left[t_{0}, t_{0}+\ln 4\right]$ because $\Phi(t, x) \leq T(t, x) \leq \theta_{*}+\varepsilon$ for these $t$. A simple computation now shows that $S_{t} \leq S_{x x}+f(S)$ for $t \in\left[t_{0}, t_{0}+\ln 4\right]$. Hence, $S$ is a subsolution of (1.6) with $S\left(t_{0}, x\right)=T\left(t_{0}, x\right)$, and so $S \leq T$ for $t \in\left[t_{0}, t_{0}+\ln 4\right]$. But $S\left(t_{0}+\ln 4,0\right) \geq \theta_{*}+2 \varepsilon>T(t, 0)$, which is a contradiction. Therefore we need to have $f\left(\theta_{*}\right)=0$.

Next, observe that one can use scaling to replace the variation in the initial condition in (1.6) by variation in the reaction strength. If $T$ solves (1.6) with $T(0, x) \equiv \chi_{[-L, L]}(x)$, define $\tilde{T}(t, x) \equiv T\left(L^{2} t, L x\right)$, so that we have

$$
\tilde{T}_{t}=\tilde{T}_{x x}+L^{2} f(\tilde{T})
$$

and $\tilde{T}(0, x)=\chi_{[-1,1]}(x)$. Hence, Theorem 1 will be proved if we show that its conclusion holds for the $L$-dependent family of problems

$$
\begin{aligned}
& T_{t}=T_{x x}+L f(T), \\
& T_{0}(x) \equiv \chi_{[-1,1]}(x)
\end{aligned}
$$

instead of (1.6) (note that Lemma 3 holds here, too). This important observation motivates the following key lemma.

Lemma 4. Let $\Omega$ be a connected open domain in $\mathbb{R}^{n}$ with a smooth boundary $\left(\right.$ possibly $\Omega=\mathbb{R}^{n}$ ) and let $f, g:[0, \infty) \rightarrow \mathbb{R}$ be Lipschitz with $f(0)=g(0)=0$ and $f \leq g$. Let $T, S:[0, \infty) \times \Omega \rightarrow[0, \infty)$ be continuous functions solving

$$
\begin{aligned}
& T_{t}=\Delta T+f(T), \\
& S_{t}=\Delta S+g(S)
\end{aligned}
$$

in $\Omega$ with Dirichlet boundary conditions on $\partial \Omega$. Assume $0 \leq T(0, x) \leq S(0, x)$ for all $x \in \Omega$ and $T\left(0, x_{0}\right)<S\left(0, x_{0}\right)$ for some $x_{0}$. Assume also that for any $\theta>0$ the set $\Omega_{0, \theta} \equiv\{x \in \Omega \mid S(0, x) \geq \theta\}$ is compact. Finally, assume that there are $\theta_{1}>0$ and $\varepsilon_{1}>0$ such that for any $\theta \in\left[\theta_{1},\|T\|_{\infty}\right)$ and $\varepsilon \in\left[0, \varepsilon_{1}\right]$ we have

$$
g\left(\theta+\varepsilon\left[\theta-\theta_{1}\right]\right) \geq(1+\varepsilon) f(\theta) .
$$

Then

$$
\liminf _{t \rightarrow \infty} \inf _{T(t, x)>\theta_{1}} \frac{S(t, x)-\theta_{1}}{T(t, x)-\theta_{1}}>1
$$

with the convention that the infimum over an empty set is $\infty$.

Remark. The result holds without change when we add a first-order term $u(x) \cdot \nabla$, with $u$ a Lipschitz vector field, to (2.2) and (2.3).

Proof. First notice that the assumptions imply that

$$
\Omega_{t, \theta} \equiv\{x \in \Omega \mid S(t, x) \geq \theta\}
$$

are compact. Indeed, by the maximum principle, $\Omega_{t, \theta} \subseteq \tilde{\Omega}_{t, \delta \theta}$ where $\delta \equiv e^{-t c}$ with $c$ the Lipschitz constant for $g$, and $\tilde{\Omega}_{t, \theta}$ is defined as $\Omega_{t, \theta}$ but with $\Phi$, the solution of

$$
\Phi_{t}=\Delta \Phi, \quad \Phi(0, x)=S(0, x),
$$

in place of $S$. Compactness of $\tilde{\Omega}_{t, \theta}$ follows from that of $\tilde{\Omega}_{0, \theta}$ and the Feynman-Kac formula. 
The assumptions and the strong maximum principle also imply $T(t, x)<S(t, x)$ for $t>0$ and $x \in \Omega$. Let us define

$$
\begin{aligned}
\Omega(t) & \equiv\left\{x \in \Omega \mid T(t, x)>\theta_{1}\right\}, \\
\Omega^{\prime}(t) & \equiv\left\{x \in \Omega \mid T(t, x)=\theta_{1}\right\},
\end{aligned}
$$

and let

$$
\omega(t) \equiv \min \left\{1+\varepsilon_{1}, \inf _{x \in \Omega(t)} \frac{S(t, x)-\theta_{1}}{T(t, x)-\theta_{1}}\right\} .
$$

Since $\overline{\Omega(t)}$ is compact and $T<S$ continuous, $\omega(t)>1$ for $t>0$. Hence the result will follow if we show that $\omega$ is a non-increasing function. Since $\omega$ is continuous (because $T$ and $S$ are), it is sufficient to show that for any $t_{0}>0$ there is $\tau_{0}>0$ such that for all $t \in\left[t_{0}, t_{0}+\tau_{0}\right]$ we have $\omega(t) \geq \omega\left(t_{0}\right)$.

Hence, fix $t_{0}>0$. Notice that the maximum principle and Feynman-Kac formula show that $\Lambda_{t_{0}, \theta} \equiv \bigcup_{t \leq t_{0}+1} \Omega_{t, \theta}$ is bounded for each $\theta>0$. Since $T, S$ are continuous, they are uniformly continuous on $\left[0, t_{0}+1\right] \times \overline{\Lambda_{t_{0}, \theta}}$, and obviously $|S(t, x)-S(s, y)| \leq$ $\theta$ and $|T(t, x)-T(s, y)| \leq \theta$ for $(t, x),(s, y) \in\left[0, t_{0}+1\right] \times\left(\mathbb{R} \backslash \Lambda_{t_{0}, \theta}^{\text {int }}\right)$. It follows that $T, S$ are uniformly continuous on $\left[0, t_{0}+1\right] \times \Omega$. Notice also that the set

$$
\Sigma \equiv\left\{(t, x) \mid t \in\left[t_{0}, t_{0}+1\right] \text { and } x \in \Omega(t) \cup \Omega^{\prime}(t)\right\} \subseteq\left[t_{0}, t_{0}+1\right] \times \Lambda_{t_{0}, \theta_{1}}
$$

is compact.

Thanks to the uniform continuity of $T$ we only need to consider the case $\Omega\left(t_{0}\right) \cup$ $\Omega^{\prime}\left(t_{0}\right) \neq \emptyset$ (otherwise $\Omega(t)=\emptyset$ and $\omega(t)=1+\varepsilon_{1}$ for $t$ close to $t_{0}$ ), and hence $\Sigma \neq \emptyset$. Since $S$ is continuous and $S>T$,

$$
\sigma \equiv \inf _{(t, x) \in \Sigma}\left\{S(t, x)-\theta_{1}\right\}>0 .
$$

We let $\delta \equiv \sigma / 4\left(1+\varepsilon_{1}\right)$ and define

$$
\Delta \equiv\left\{x \in \Omega|| T\left(t_{0}, x\right)-\theta_{1} \mid \leq \delta \text { and } S\left(t_{0}, x\right)-\theta_{1} \geq \sigma-\delta\right\} .
$$

By the uniform continuity of $T, S$, there is $\tau_{0} \in(0,1)$ such that for $t \in\left[t_{0}, t_{0}+\tau_{0}\right]$ and $x \in \Omega$ we have

$$
\left|T(t, x)-T\left(t_{0}, x\right)\right| \leq \frac{\delta}{2} \quad \text { and } \quad\left|S(t, x)-S\left(t_{0}, x\right)\right| \leq \frac{\delta}{2}
$$

So if $t \in\left[t_{0}, t_{0}+\tau_{0}\right]$, then

$$
\Omega(t) \subseteq \Omega\left(t_{0}\right) \cup \Delta
$$

(note that $S\left(t_{0}, x\right)-\theta_{1} \geq \sigma-\frac{\delta}{2}$ for $x \in \Omega(t)$ because then $(t, x) \in \Sigma$ ). Now if $t \in\left[t_{0}, t_{0}+\tau_{0}\right]$ and $x \in \Delta$, then by (2.6),

$$
S(t, x)-\theta_{1}>\frac{\sigma}{2}>\left(1+\varepsilon_{1}\right)\left|T(t, x)-\theta_{1}\right| \geq \omega\left(t_{0}\right)\left(T(t, x)-\theta_{1}\right) .
$$

Next let

$$
A \equiv\left\{x \in \Omega \mid T\left(t_{0}, x\right)>\theta_{1}+\delta\right\}=\Omega\left(t_{0}\right) \backslash \Delta
$$

and

$$
B \equiv\left\{x \in \Omega \mid T\left(t_{0}, x\right) \geq \theta_{1}+\frac{\delta}{2}\right\} \subseteq \Omega\left(t_{0}\right) .
$$

Uniform continuity of $T$ shows that $\operatorname{dist}\left(A, B^{c}\right)>0$, and so there is an open set $\Gamma$ with a smooth boundary such that $A \subseteq \Gamma \subseteq B$. Let $\tilde{T} \equiv T-\theta_{1}, \tilde{U} \equiv \omega\left(t_{0}\right) \tilde{T}$, $\tilde{S} \equiv S-\theta_{1}, \tilde{f}(\theta) \equiv f\left(\theta+\theta_{1}\right)$, and $\tilde{g}(\theta) \equiv g\left(\theta+\theta_{1}\right)$. Then for $x \in \Gamma$ we have

$$
\tilde{S}\left(t_{0}, x\right) \geq \omega\left(t_{0}\right) \tilde{T}\left(t_{0}, x\right)=\tilde{U}\left(t_{0}, x\right)
$$


by the definition of $\omega\left(t_{0}\right)$. For $t \in\left[t_{0}, t_{0}+\tau_{0}\right]$ and $x \in \partial \Gamma$ we have

$$
\tilde{S}(t, x)>\sigma-2 \delta>\frac{\sigma}{2} \geq \omega\left(t_{0}\right) \tilde{T}(t, x)=\tilde{U}(t, x)
$$

since $\partial \Gamma \subseteq B \backslash A \subseteq \Delta$. Also, for $t \in\left[t_{0}, t_{0}+\tau_{0}\right]$ and $x \in \Gamma$ we have

$$
\begin{aligned}
\tilde{U}_{t} & =\Delta \tilde{U}+\omega\left(t_{0}\right) \tilde{f}\left(\frac{1}{\omega\left(t_{0}\right)} \tilde{U}\right), \\
\tilde{S}_{t} & =\Delta \tilde{S}+\tilde{g}(\tilde{S})
\end{aligned}
$$

by (2.2) and (2.3). For these $(t, x)$ we have $T(t, x) \geq \theta_{1}$ because of (2.6) and $\Gamma \subseteq B$, and so by (2.4) and $\omega\left(t_{0}\right)-1 \in\left(0, \varepsilon_{1}\right]$,

$$
\omega\left(t_{0}\right) \tilde{f}\left(\frac{1}{\omega\left(t_{0}\right)} \tilde{U}\right)=\omega\left(t_{0}\right) f(T) \leq g\left(\omega\left(t_{0}\right)\left[T-\theta_{1}\right]+\theta_{1}\right)=\tilde{g}(\tilde{U}) .
$$

The comparison principle now shows that $\tilde{S} \geq \tilde{U}$ on $\left[t_{0}, t_{0}+\tau_{0}\right] \times \Gamma$. Hence

$$
S(t, x)-\theta_{1} \geq \omega\left(t_{0}\right)\left(T(t, x)-\theta_{1}\right)
$$

for $t \in\left[t_{0}, t_{0}+\tau_{0}\right]$ and $x \in A$, which together with (2.7), (2.8), and (2.9) gives $\omega(t) \geq \omega\left(t_{0}\right)$ for $t \in\left[t_{0}, t_{0}+\tau_{0}\right]$. The proof is finished.

\section{Proof of Theorem 1}

We can now complete the proof of Theorem 1, We will do this for the formulation in (2.1).

First assume $\theta_{0}>0$. We know from Lemma 3 (iii) that for every $L$ we have $T \rightarrow$ $\theta_{*}^{L}$ uniformly on compacts, with $\theta_{*}^{L}$ such that $f\left(\theta_{*}^{L}\right)=0$. Obviously $\theta_{*}^{L} \notin\left(0, \theta_{0}\right)$ because in that case we would have $T(t, x) \leq \theta_{0}$ for all $t \geq t_{0}$ and consequently $T \rightarrow 0$ (since $\left\|T\left(t_{0}, \cdot\right)\right\|_{1}<\infty$ and $T_{t}=T_{x x}$ for $t \geq t_{0}$ ). So we are only left with $\theta_{*}^{L} \in\left\{0, \theta_{0}, 1\right\}$.

Let $A, B$, and $C$ be the sets of $L \geq 0$ such that $\theta_{*}^{L}$ equals $0, \theta_{0}$, and 1 , respectively. Notice that since $T(t, 0) \geq T(t, x)$, the convergence of $T$ to 0 for $L \in A$ is actually uniform on $\mathbb{R}$. We have $A \cup B \cup C=[0, \infty)$, and the comparison principle implies that the three sets are intervals with $A$ lying to the left of $B$ and $B$ to the left of C.

Moreover, $A$ and $C$ are non-empty by Kanel' [9] and open. The latter follows from the fact that if $T^{L}$ is the solution of (2.1), then for $L_{1}<L_{2}$ and $V \equiv T^{L_{2}}-T^{L_{1}}$ we have $V \geq 0$ by comparison, $V(0)=0$, and

$$
\begin{aligned}
V_{t} & =\Delta V+\left(L_{2}-L_{1}\right) f\left(T^{L_{2}}\right)+L_{1}\left[f\left(T^{L_{2}}\right)-f\left(T^{L_{1}}\right)\right] \\
& \leq \Delta V+c\left(L_{2}-L_{1}\right)+c L_{1} V
\end{aligned}
$$

with $c \geq\|f\|_{\infty}$ the Lipschitz constant for $f$. Since the function

$$
\tilde{V}(t, x) \equiv \frac{L_{2}-L_{1}}{L_{1}}\left(e^{c L_{1} t}-1\right)
$$

(or $\tilde{V}(t, x) \equiv c L_{2} t$ when $L_{1}=0$ ) satisfies

$$
\tilde{V}_{t}=\Delta \tilde{V}+c\left(L_{2}-L_{1}\right)+c L_{1} \tilde{V}
$$

with $\tilde{V}(0)=0$, the comparison principle gives $V \leq \tilde{V}$, that is,

$$
T^{L_{2}}(t, x)-T^{L_{1}}(t, x) \in\left[0, \frac{L_{2}-L_{1}}{L_{1}}\left(e^{c L_{1} t}-1\right)\right] .
$$

Therefore if $L_{1} \in A$, then $T^{L_{1}}\left(t_{0}, 0\right) \leq \frac{1}{2} \theta_{0}$ for some $t_{0}>0$, and hence $T^{L_{2}}\left(t_{0}, 0\right)<$ $\theta_{0}$ (and so $\left.L_{2} \in A\right)$ for $L_{2}<L_{1}+\frac{1}{2} \theta_{0} L_{1}\left(e^{c L_{1} t_{0}}-1\right)^{-1}$. On the other hand, Kanel's result [9] also holds for (1.5), and it says that for any $\alpha>\theta_{0}$ and $L>0$ there 
is $M=M(\alpha, L)<\infty$ such that if $T$ solves (2.1) and $T\left(t_{0}, x\right) \geq \alpha \chi_{[-M, M]}(x)$, then $T \rightarrow 1$ uniformly on compacts. Let $\theta_{0}<\alpha<\beta<1$ and if $L_{1} \in C$, let $M=M\left(\alpha, \frac{1}{2} L_{1}\right)$. For some $t_{0}$ we have $T^{L_{1}}\left(t_{0}, x\right) \geq \beta \chi_{[-M, M]}(x)$ and so for any $L_{2}>L_{1}-(\beta-\alpha) L_{1}\left(e^{c L_{1} t_{0}}-1\right)^{-1}$ we have $T^{L_{2}}\left(t_{0}, x\right) \geq \alpha \chi_{[-M, M]}(x)$. If in addition $L_{2}>\frac{1}{2} L_{1}$, we have $L_{2} \in C$. So $A, C$ are non-empty and open, and hence $B$ is nonempty and closed.

The proof will be finished if we show that $B$ contains a single element. Hence assume $L_{1}<L_{2}$ are both in $B$. Let $\theta_{1} \equiv \frac{1}{2} \theta_{0} \in\left(0, \theta_{0}\right)$ and

$$
\varepsilon_{1} \equiv \min \left\{L_{2} L_{1}^{-1}-1, \delta\left(\delta+\theta_{0}\right)^{-1}\right\}>0
$$

(with $\delta$ from the statement of Theorem 1). Choose $t_{0}>0$ such that $T^{L_{1}}(t, x) \leq$ $\theta_{0}+\frac{\delta}{2}$ when $t \geq t_{0}$. The comparison principle, $f \not \equiv 0$, and the strong maximum principle yield $T^{L_{1}}<T^{L_{2}}$ for $t>0$ and both $T^{L_{1}}$ and $T^{L_{2}}$ are obviously continuous for $t>0$. Lipschitzness of $f$ and compact support of $T^{L_{2}}(0, \cdot)$ show that for any $\theta>0$, the set of $x$ for which $T^{L_{2}}\left(t_{0}, x\right) \geq \theta$ is compact. Finally, whenever $\theta \in\left[\theta_{1}, \theta_{0}+\frac{\delta}{2}\right]$ and $\varepsilon \in\left[0, \varepsilon_{1}\right]$, we have $\theta+\varepsilon\left[\theta-\theta_{1}\right] \leq \theta_{0}+\delta$. Thus by the assumptions on $f$ (and the definition of $\varepsilon_{1}$ ),

$$
L_{2} f\left(\theta+\varepsilon\left[\theta-\theta_{1}\right]\right) \geq L_{2} f(\theta) \geq(1+\varepsilon) L_{1} f(\theta) .
$$

Therefore Lemma 4 applies to $T^{L_{1}}$ and $T^{L_{2}}$ (with starting time $t_{0}$ ) and shows that for some $r>1$ and all large enough $t$ we have

$$
T^{L_{2}}(t, x)-\theta_{1} \geq r\left[T^{L_{1}}(t, x)-\theta_{1}\right]
$$

whenever $T^{L_{1}}(t, x)>\theta_{1}$. But since $\theta_{1}<\theta_{0}$, this contradicts the assumption that both $T^{L_{1}}(t, 0)$ and $T^{L_{2}}(t, 0)$ converge to $\theta_{0}$ as $t \rightarrow \infty$. Hence, $B=\left\{L_{0}\right\}$ and we are done with the case $\theta_{0}>0$.

Now, consider $\theta_{0}=0$. We have $\theta_{*}^{L} \in\{0,1\}$, the sets $A, C$ satisfy $A \cup C=[0, \infty)$, and by the comparison principle, $A$ lies to the left of $C$. Moreover, $0 \in A$ and $C$ is non-empty and open by the same argument as above. Hence $A$ is closed and its maximum is $L_{0}$ (possibly $L_{0}=0$ ). Lemma 3(iii) yields (iii) of this theorem and $T(t, 0) \geq T(t, x)$ gives (i) and (ii), including the fact that the convergence in (ii) is uniform on $\mathbb{R}$. The proof is finished.

\section{Proof of Theorem 2}

The situation is somewhat more complicated here. Firstly, we do not have Lemma 3(iii) at our disposal, and so the limit of $T$ as $t \rightarrow \infty$ need not always be a constant function. Secondly, we cannot use Lemma 4 and the scaling argument preceding it in the way we did in the last section because it is no longer true that $L_{2} f \geq L_{1} f$ when $L_{2}>L_{1}$. We note that one can still use the lemma without scaling, but then the argument applies only to a restricted class of bistable $f$. Fortunately, it turns out that the first of these difficulties actually cancels the problems created by the second, as we shall see below.

Let us therefore go back to $T$ solving (1.6) rather than (2.1). We know from Lemma 3(ii) that $\theta_{*}^{L} \equiv \lim _{t \rightarrow \infty} T(t, 0)$ is well defined, and from the comparison principle that it is non-decreasing in $L$.

First assume $\theta_{*}^{L}<\theta_{2}$, with $\theta_{2}$ defined in the introduction by $\int_{0}^{\theta_{2}} f(\theta) d \theta=0$. Choose $\varepsilon>0$ and a Lipschitz function $\tilde{f}:[0,1] \rightarrow \mathbb{R}$ so that $\tilde{f}=0$ on $[0, \varepsilon]$, 
$\tilde{f}^{\prime}(\varepsilon)<0, \tilde{f} \geq f$ on $\left(\varepsilon, \frac{1}{2}\left(\theta_{*}^{L}+\theta_{2}\right)\right]$ and $\tilde{f}$ has a single zero there, $\tilde{f}>0$ on $\left(\frac{1}{2}\left(\theta_{*}^{L}+\theta_{2}\right), 1\right), \tilde{f}(1)=0>\tilde{f}^{\prime}(1)$, and

$$
\int_{0}^{1} \tilde{f}(\theta) d \theta<0 .
$$

Let $t_{0}$ be such that for $t \geq t_{0}$ and all $x \in \mathbb{R}$ we have $T(t, x) \leq \frac{1}{2}\left(\theta_{*}^{L}+\theta_{2}\right)$. This is possible by Lemma $3(\mathrm{i})$. Since $\tilde{f} \geq f$ on $\left[0, \frac{1}{2}\left(\theta_{*}^{L}+\theta_{2}\right)\right]$, starting from time $t_{0}$ one has $T_{t} \leq T_{x x}+\tilde{f}(T)$, that is, $T$ is a subsolution of the equation

$$
\Phi_{t}=\Phi_{x x}+\tilde{f}(\Phi) .
$$

Let $\phi: \mathbb{R} \rightarrow[0,1]$ with $\phi(x) \rightarrow \varepsilon$ as $x \rightarrow \infty$ and $\phi(x) \rightarrow 1$ as $x \rightarrow-\infty$ be the unique, up to translation, traveling front profile (with speed $v$ ) for (4.2) [8]. That is, $\phi(x-v t)$ solves (4.2). It follows from (4.1) that in this case $v<0$.

From compactness of the support of $T(0, x)$ and Lipschitzness of $f, T\left(t_{0}, x\right) \rightarrow 0$ as $|x| \rightarrow \infty$. This and $\left\|T\left(t_{0}, \cdot\right)\right\|_{\infty}<1$ mean that there is $x_{0}$ such that $T\left(t_{0}, x\right) \leq$ $\phi\left(x-x_{0}-v t_{0}\right)$, and since $\phi\left(x-x_{0}-v t\right)$ is a solution and $T(t, x)$ a subsolution of (4.2),

$$
T(t, x) \leq \phi\left(x-x_{0}-v t\right)
$$

for all $t \geq t_{0}$. But then $T(t, 0) \leq \phi\left(-x_{0}-v t\right) \rightarrow \varepsilon$ as $t \rightarrow \infty$ because $v<0$. This holds for any $\varepsilon>0$ and thus $\theta_{*}^{L}=0$.

Next assume $\theta_{*}^{L}>\theta_{2}$. Let $S$ be the solution of (1.6) on $\mathbb{R}^{+}$with $S(0, x)=0$ and $S(t, 0)=s(t)$ a smooth strictly increasing function with all derivatives bounded such that $s(0)=0, s(t) \leq T(t, 0)$, and $\lim _{t \rightarrow \infty} s(t)=\theta_{*}^{L}$. Then for any $h>0$ we have $S(h, x)>S(0, x)$ and so by comparison $S(t+h, x)>S(t, x)$. Hence $\tilde{S}(x) \equiv \lim _{t \rightarrow \infty} S(t, x)>0$ is well defined and $\tilde{S}(0)=\theta_{*}^{L}$. Since by comparison again, $S(t, x) \leq T(t, x) \leq T(t, 0)$, we also have $\tilde{S}(x) \leq \theta_{*}^{L}$.

Standard parabolic regularity shows that $S(t, x)$ converges to $\tilde{S}(x)$ along with its first two derivatives uniformly on compacts, and so $\tilde{S}$ solves the stationary problem

$$
0=\tilde{S}^{\prime \prime}+f(\tilde{S})
$$

on $\mathbb{R}^{+}$(this can be found also in [1]). But then for any $y>0$,

$$
\int_{\tilde{S}(y)}^{\theta_{*}^{L}} f(\theta) d \theta=\int_{y}^{0} f(\tilde{S}(x)) \tilde{S}^{\prime}(x) d x=\int_{0}^{y} \tilde{S}^{\prime \prime}(x) \tilde{S}^{\prime}(x) d x=\frac{1}{2}\left[\left(\tilde{S}^{\prime}(y)\right)^{2}-\left(\tilde{S}^{\prime}(0)\right)^{2}\right] .
$$

Assume there is $z>0$ such that $\tilde{S}(z)<\theta_{*}^{L}$, and then pick one such that also $\tilde{S}^{\prime}(z)<0$. Since $\int_{w}^{\theta_{*}^{L}} f(\theta) d \theta$ is bounded below by a positive constant for all $w \in$ $[0, \tilde{S}(z)]$, the same must be true for $\left(\tilde{S}^{\prime}(y)\right)^{2}$ when $\tilde{S}(y) \in[0, \tilde{S}(z)]$. But $\tilde{S}^{\prime}(z)<0$, continuity of $\tilde{S}^{\prime}$, and $\tilde{S}>0$ now imply that $\tilde{S}$ is decreasing and positive on $[z, \infty)$, with $\tilde{S}^{\prime}$ bounded away from zero - a contradiction. Hence, we must have $\tilde{S} \equiv \theta_{*}^{L}$, which is only possible if $\theta_{*}^{L}=1$. Moreover, since $S$ converges to $\tilde{S} \equiv 1$ uniformly on compacts as $t \rightarrow \infty$ (and $S \leq T \leq 1$ ), so does $T$.

The above shows that $\theta_{*}^{L} \in\left\{0, \theta_{2}, 1\right\}$. As in the proof of Theorem 11, and using the equivalent of Kanel"s result for (1.5) and bistable $f$ [6], one can show that the intervals $A, C$ of $L$ for which $\theta_{*}^{L}=0,1$, respectively, are non-empty and open. If $B$ is the closed interval of $L$ for which $\theta_{*}^{L}=\theta_{2}$, then $B$ lies between $A$ and $C$ and again $A \cup B \cup C=[0, \infty)$. 
Next we need to prove that $B$ only contains one element. We will show below that if $L \in B$, then $T(t, x) \rightarrow U(x)$ uniformly on $\mathbb{R}$ as $t \rightarrow \infty$. Here $U$ solves (4.3) with $U(0)=\theta_{2}$ and $U^{\prime}(0)=0$. Assume now that $L_{1}<L_{2}$ are both in $B$, with $T^{L_{1}}$ and $T^{L_{2}}$ the corresponding solutions of (1.6). We then have $T^{L_{1}}(t, 0) \rightarrow \theta_{2}$, and since the equation is translation invariant, we also have $\tilde{T}(t, \varepsilon) \rightarrow \theta_{2}$ when $\tilde{T}$ solves (1.6) with initial condition $\tilde{T}_{0}(x) \equiv \chi_{\left[-L_{1}+\varepsilon, L_{1}+\varepsilon\right]}(x)$. But if $|\varepsilon|<L_{2}-L_{1}$, then $T_{0}^{L_{2}}(x) \geq \tilde{T}_{0}(x)$, and so by the comparison principle,

$$
U(\varepsilon)=\lim _{t \rightarrow \infty} T^{L_{2}}(t, \varepsilon) \geq \lim _{t \rightarrow \infty} \tilde{T}(t, \varepsilon)=\theta_{2} .
$$

Since $U^{\prime \prime}(0)=-f(U(0))=-f\left(\theta_{2}\right)<0, U$ has a strict local maximum at zero and therefore $U(\varepsilon)<U(0)=\theta_{2}$ for all small enough $|\varepsilon|>0$. This is a contradiction and hence $B=\left\{L_{0}\right\}$.

To complete the proof, we need to show that $T(t, x) \rightarrow U(x)$ uniformly as $t \rightarrow \infty$ when $L \in B$ (and hence $\theta_{*}^{L}=\theta_{2}$ ). Notice that the argument following (4.3) applies to $U$ and we find for any $x>0$ such that $U(x) \geq 0$,

$$
\int_{U(x)}^{\theta_{2}} f(\theta) d \theta=\frac{1}{2}\left(U^{\prime}(x)\right)^{2} .
$$

The definition of $\theta_{2}$ then shows that $U(x) \leq \theta_{2}$, and $U^{\prime}(x) \neq 0$ when $U(x) \in\left(0, \theta_{2}\right)$. Since $U(x)$ cannot equal the constant $\theta_{2}$ on any interval and $U^{\prime}$ is continuous, we must have $U^{\prime}(x)<0$ for all $x>0$ such that $U(x)>0$. There is no $x$ with $U(x)=0$ because then (4.4) would give $U^{\prime}(x)=0$, contradicting uniqueness of solutions to initial value problems associated to (4.3). Hence $U(x) \in\left(0, \theta_{2}\right)$ and $U^{\prime}(x)<0$ for $x>0$, with $U^{\prime}(x)$ bounded away from zero when $U(x)$ is away from zero (by (4.4) and the definition of $\theta_{2}$ ). This and symmetry show that $U$ is indeed a symmetric bell-shaped solution (with $U^{\prime}$ decreasing on $\left[0, U^{-1}\left(\theta_{0}\right)\right]$ and increasing on $\left[U^{-1}\left(\theta_{0}\right), \infty\right)$ by (4.4)) of the stationary problem (4.3) such that $U(x) \rightarrow 0$ as $|x| \rightarrow \infty$.

If we now apply the argument involving $S$ and $\tilde{S}$ from the case $\theta_{*}^{L}>\theta_{2}$, we find as above that $\tilde{S}(x) \leq \theta_{2}=\tilde{S}(0)$ and that $\tilde{S}>0$ is possible only if $\tilde{S}^{\prime}(0)=0$. But then $\tilde{S}(0)=U(0)$ and $\tilde{S}^{\prime}(0)=U^{\prime}(0)$; thus $\tilde{S}=U$. Moreover, uniform on compacts convergence of $S$ to $U$ and $0 \leq S(t, x) \leq U(x) \rightarrow 0$ as $|x| \rightarrow \infty$ yield uniform on $\mathbb{R}$ convergence of $S$ to $U$. Since $T(t, x) \geq S(t, x)$, we have $\liminf _{t \rightarrow \infty} T(t, x) \geq U(x)$ uniformly on $\mathbb{R}$. Here "uniformly on $\mathbb{R}$ " means that for every $\varepsilon>0$ there is $\tau<\infty$ so that $T(t, x) \geq U(x)-\varepsilon$ for any $t>\tau$ and $x \in \mathbb{R}$. Hence we are left with proving $\lim \sup _{t \rightarrow \infty} T(t, x) \leq U(x)$ uniformly in $x>0$ (which suffices due to symmetry).

Let $0<x_{0}<\infty$ be such that if $S(0, x) \geq \theta_{2} \chi_{\left[-x_{0}, x_{0}\right]}(x)$ and $S$ satisfies (1.6), then $S \rightarrow 1$ uniformly on compacts. Such $x_{0}$ exists by [6] because $\theta_{2}>\theta_{0}$. Then obviously for every $t \geq 0$ we have $T\left(t, x_{0}\right) \leq \theta_{2}$, because otherwise Lemma 33(i) would imply $T \rightarrow 1$ uniformly on compacts. Since both $T(t, x)$ and $V(x)=$ $U\left(x-x_{0}\right)$ satisfy (1.6) on $\left(x_{0}, \infty\right), V\left(x_{0}\right)=\theta_{2} \geq T\left(t, x_{0}\right)$, and $V(x)>0=T(0, x)$ for $x>x_{0}$, the comparison principle implies $T(t, x) \leq V(x)$. Let us therefore define

$$
x_{1} \equiv \min \left\{\tilde{x} \mid \limsup _{t \rightarrow \infty} T(t, x) \leq U(x-\tilde{x}) \text { uniformly in } x>\tilde{x}\right\} \leq x_{0} .
$$

The minimum is achieved because $U$ is uniformly continuous and by Lemma 3(i), $T(t, x) \leq T(t, 0) \rightarrow \theta_{2}=U(0)$. We note that at this point one can derive $T \rightarrow U$ from $x_{1}<\infty$ and the results of [3] if $f \in C^{1}(0,1)$ and $f^{\prime}(0)<0$. However, our non-linearity is more general and so [3] is not applicable here. 
If $x_{1}=0$, then we are done, so assume $x_{1}>0$. First notice that

$$
\limsup _{t \rightarrow \infty} T\left(t, \frac{1}{2} x_{1}\right) \leq \theta_{2}-\delta_{1}
$$

for some $\delta_{1}>0$. Indeed, in view of $\lim _{t \rightarrow \infty} T(t, 0)=\theta_{2}$, Lemma 3(i), and the comparison principle, it is sufficient to show that there are $\delta_{1}, \delta_{2}>0$ such that if $S(0, x) \geq\left(\theta_{2}-\delta_{1}\right) \chi_{\left[-x_{1} / 2, x_{1} / 2\right]}(x)$ and $S$ satisfies (1.6), then $S(t, 0) \geq \theta_{2}+\delta_{2}$ for some $t>0$. This in turn is true because it holds for $\delta_{1}=0$ and some $t, \delta_{2}>0$, since then $S(0,0)=\theta_{2}$ and $S_{t}(0,0)=f\left(\theta_{2}\right)>0$, and because $S(t, 0)$ is continuous in $\delta_{1}$.

Now choose $x_{2} \in\left(\frac{1}{2} x_{1}, x_{1}\right)$ such that $U\left(x_{1}-x_{2}\right) \geq \theta_{2}-\delta_{1}$. The above and Lemma $3(\mathrm{i})$ show that $\limsup _{t \rightarrow \infty} T(t, x) \leq \theta_{2}-\delta_{1}$ uniformly in $x \geq \frac{1}{2} x_{1}$, and so

$$
\limsup _{t \rightarrow \infty} T(t, x) \leq U\left(x-x_{2}\right)
$$

uniformly in $x \in\left[x_{2}, x_{1}\right]$. We will show that (4.5) also holds uniformly in $x>x_{1}$, which will yield $x_{1} \leq x_{2}$ by the definition of $x_{1}$. This will be a contradiction and hence necessarily $x_{1}=0$.

Let $s(t)$ be smooth, decreasing, with all derivatives bounded, such that $s(0)=\theta_{2}$ and $\lim _{t \rightarrow \infty} s(t)=U\left(x_{1}-x_{2}\right)$. Let $S(t, x)$ satisfy (1.6) for $x>x_{1}$ with $S(0, x)=$ $U\left(x-x_{1}\right)$ and $S\left(t, x_{1}\right)=s(t)$. As above, one proves that this time $S$ is nonincreasing in $t$,

$$
S(t, x) \in\left[U\left(x-x_{2}\right), U\left(x-x_{1}\right)\right],
$$

and $\tilde{S}(x) \equiv \lim _{t \rightarrow \infty} S(t, x)$ satisfies (4.3) with $\tilde{S}(x) \rightarrow 0$ as $x \rightarrow \infty$ (by (4.6)). Moreover, $S \rightarrow \tilde{S}$ uniformly on compacts, which together with (4.6) and $U(x) \rightarrow 0$ as $x \rightarrow \infty$ shows that $S \rightarrow \tilde{S}$ uniformly on $\mathbb{R}$. Since $\tilde{S}\left(x_{1}\right)=U\left(x_{1}-x_{2}\right)$ and $\tilde{S}(\infty)=U(\infty)=0$, a formula similar to (4.4), with the integral from 0 to $U\left(x_{1}-x_{2}\right)$, gives $\tilde{S}^{\prime}\left(x_{1}\right)=U^{\prime}\left(x_{1}-x_{2}\right)$. It follows that $\tilde{S}(x)=U\left(x-x_{2}\right)$.

Now pick any $\varepsilon>0$ and choose $t_{0}$ such that

$$
S(t, x)-U\left(x-x_{2}\right)<\frac{\varepsilon}{2}
$$

for $t \geq t_{0}$ and $x \geq x_{1}$. Then pick $t_{1}$ so that

$$
T(t, x)-U\left(x-x_{1}\right)<\varepsilon_{0} \equiv \frac{\varepsilon}{2} e^{-c t_{0}}
$$

for $t \geq t_{1}$ and $x \geq x_{1}$ (with $c$ the Lipschitz constant for $f$ ). This is possible by the definition of $x_{1}$.

For any $t_{2}>t_{1}$ and $x>x_{1}$ we have

$$
T\left(t_{2}, x\right)-S(0, x)=T\left(t_{2}, x\right)-U\left(x-x_{1}\right)<\varepsilon_{0},
$$

and for $t>t_{2}$ we have

$$
T\left(t, x_{1}\right)-S\left(t-t_{2}, x_{1}\right)=T\left(t, x_{1}\right)-s\left(t-t_{2}\right) \leq T\left(t, x_{1}\right)-U\left(x_{1}-x_{2}\right)<\varepsilon_{0}
$$

by (4.5) if $t_{2}$ is large enough. Hence if we let $R(t, x) \equiv S(t, x)+e^{c t} \varepsilon_{0}$, then $T\left(t_{2}, x\right)<R(0, x)$ for $x>x_{1}, T\left(t, x_{1}\right)<R\left(t-t_{2}, x_{1}\right)$ for $t>t_{2}$, and

$$
R_{t}=S_{t}+c e^{c t} \varepsilon_{0}=S_{x x}+f(S)+c e^{c t} \varepsilon_{0} \geq R_{x x}+f(R) .
$$


So $R$ is a supersolution of (1.6), and by the comparison principle, $T(t, x) \leq$ $R\left(t-t_{2}, x\right)$ for $t>t_{2}$ and $x>x_{1}$. In particular,

$$
T\left(t_{2}+t_{0}, x\right) \leq R\left(t_{0}, x\right)=S\left(t_{0}, x\right)+\frac{\varepsilon}{2}<U\left(x-x_{2}\right)+\varepsilon
$$

for $x>x_{1}$ by (4.7). Since this holds for any large enough $t_{2}$, we have $T(t, x)<$ $U\left(x-x_{2}\right)+\varepsilon$ for all large $t$ and $x>x_{1}$. As $\varepsilon>0$ was arbitrary, this gives (4.5) uniformly in $x>x_{1}$. Hence $x_{1} \leq x_{2}<x_{1}$, a contradiction. Therefore we must have $x_{1}=0$ and the proof is finished.

\section{ACKNOWLEDGMENTS}

The author thanks Henri Berestycki, Peter Constantin, François Hamel, Alexander Kiselev, Peter Poláčik, Jean-Michel Roquejoffre, and Lenya Ryzhik for encouragement and useful discussions. He also acknowledges the hospitality of the Mathematics Department of the University of Chicago where part of this work was done, as well as partial support from the NSF through the grant DMS-0314129.

\section{REFERENCES}

[1] D.G. Aronson and H.F. Weinberger, Nonlinear diffusion in population genetics, combustion, and nerve pulse propagation, Partial differential equations and related topics, Lecture Notes in Math. 446, Springer, Berlin, 1975. MR0427837(55:867)

[2] H. Berestycki, The influence of advection on the propagation of fronts in reaction-diffusion equations, Nonlinear PDEs in Condensed Matter and Reactive Flows, NATO Science Series C, 569, H. Berestycki and Y. Pomeau eds., Kluwer, Dordrecht, 2003.

[3] J. Busca, M.A. Jendoubi, and P. Poláčik, Convergence to equilibrium for semilinear parabolic problems in $\mathbb{R}^{N}$, Comm. Partial Differential Equations 27 (2002), 1793-1814. MR 1941658 (2003m:35110)

[4] P. Constantin, A. Kiselev, and L. Ryzhik, Quenching of flames by fluid advection, Comm. Pure Appl. Math. 54 (2001), 1320-1342. MR1846800 (2002f:35138)

[5] A. Fannjiang, A. Kiselev, and L. Ryzhik, Quenching of reaction by cellular flow, preprint.

[6] P.C. Fife and J.B. McLeod, The approach of solutions of nonlinear diffusion equations to travelling front solutions, Arch. Ration. Mech. Anal. 65 (1977), 335-361. MR0442480 (56:862)

[7] R.A. Fisher, The advance of advantageous genes, Ann. of Eugenics 7 (1937), 355-369.

[8] Ja.I. Kanel', Stabilization of solutions of the Cauchy problem for equations encountered in combustion theory, Mat. Sb. (N.S.) 59 (101) 1962 suppl., 245-288. MR0157130 (28:367)

[9] Ja.I. Kanel', Stabilization of the solutions of the equations of combustion theory with finite initial functions, Mat. Sb. (N.S.)65 (107) 1964 suppl., 398-413. MR0177209(31:1473)

[10] A. Kiselev and A. Zlatoš, Quenching of combustion by shear flows, to appear in Duke Math. $\mathrm{J}$.

[11] A.N. Kolmogorov, I.G. Petrovskii, and N.S. Piskunov, Étude de l'équation de la chaleur de matière et son application à un problème biologique, Bull. Moskov. Gos. Univ. Mat. Mekh. 1 (1937), 1-25.

[12] J. Nagumo, S. Arimoto, and S. Yoshizawa, An active pulse transmission line simulating nerve axon, Proc. Inst. Radio Eng. 50 (1962), 2061-2070.

[13] J.-M. Roquejoffre, Eventual monotonicity and convergence to travelling fronts for the solutions of parabolic equations in cylinders, Ann. Inst. H. Poincaré Anal. Non Linéaire 14 (1997), 499-552. MR1464532 (98h:35107)

[14] J. Smoller, Shock Waves and Reaction-Diffusion Equations, Springer-Verlag, New York, 1994. MR:1301779 (95g:35002)

[15] J. Xin, Existence and nonexistence of travelling waves and reaction-diffusion front propagation in periodic media, J. Stat. Phys. 73 (1993), 893-926. MR.1251222 (94k:35155)

[16] J. Xin, Front propagation in heterogeneous media, SIAM Rev. 42 (2000), 161-230. MR1778352(2001i:35184) 
[17] J.B. Zel'dovich and D.A. Frank-Kamenetskii, A theory of thermal propagation of flame, Acta Physiochimica USSR 9 (1938), 341-350.

[18] A. Zlatoš, Quenching and propagation of combustion without ignition temperature cutoff, Nonlinearity 18 (2005), 1463-1475.

Department of Mathematics, University of Wisconsin, Madison, Wisconsin 53706

E-mail address: zlatos@math.wisc.edu 